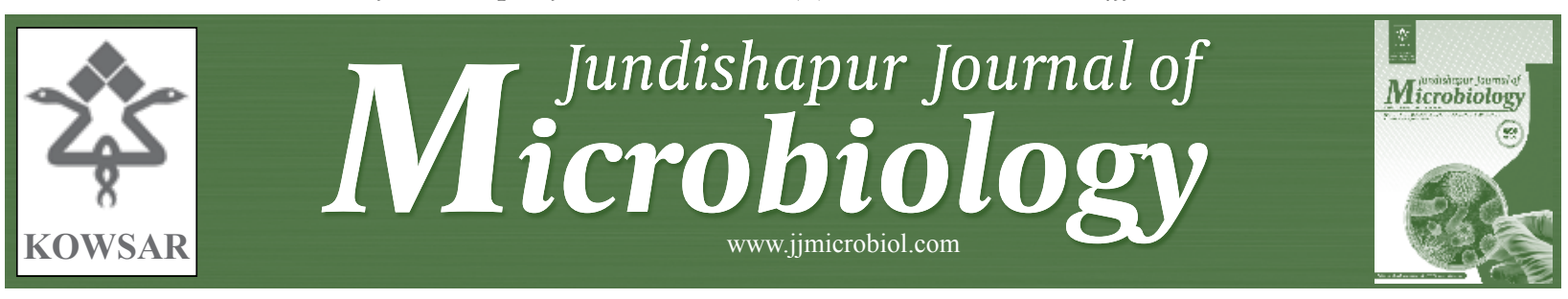

\title{
PerR-Mediated Oxidative Stress Response in Staphylococcus aureus
}

\author{
Judy Y. Ikawa Wakabayashi ${ }^{1}$, Davis W. Cheng ${ }^{1,2^{*}}$ \\ ${ }^{1}$ Department of Biology, California State University, Fresno, California, USA \\ ${ }^{2}$ Medical Research Infrastructure for Minority Institutions, California State University, Fresno, California, USA
}

\begin{tabular}{|c|c|}
\hline A R T I C L E I N F O & A B $S T$ T A C T \\
\hline $\begin{array}{l}\text { Article type: } \\
\text { Review Article }\end{array}$ & $\begin{array}{l}\text { Staphylococcus aureus is a human bacterial pathogen, known to cause a variety of illness- } \\
\text { es and infections in the hospital and community settings. It produces a large number of } \\
\text { virulence factors used for host invasion, tissue colonization and destruction, leading to }\end{array}$ \\
\hline $\begin{array}{l}\text { Article history: } \\
\text { Received: } 02 \text { Oct } 2011 \\
\text { Revised: } 27 \text { Dec } 2011 \\
\text { Accepted: } 04 \text { Jan } 2012\end{array}$ & $\begin{array}{l}\text { severe infections in both healthy and immunodeficient people around the world. In this } \\
\text { paper, we studied the potential mechanisms related to methicillin resistant } S \text {. aureus } \\
\text { (MRSA) infection, analyzed the significance of oxidative stress for survival of S. aureus, } \\
\text { and re-identified the molecular structure, regulatory boxes, and regulons of PerR (perox- }\end{array}$ \\
\hline $\begin{array}{l}\text { Keywords: } \\
\text { Bacillus subtilis } \\
\text { Manganese } \\
\text { Iron }\end{array}$ & $\begin{array}{l}\text { investigated how PerR functions in response to oxidative stress and how the PerR regu- } \\
\text { lon affects } S \text {. aureus virulence. As a conclusion, we postulate that the regulation of PerR } \\
\text { and its regulon indirectly influences the expression of virulence factors by prolonging } \\
\text { the pathogen's survival under harsh conditions that may lead to the increased risk of } S \text {. } \\
\text { aureus transmission, infection, and spread of the disease. }\end{array}$ \\
\hline
\end{tabular}

Copyright $\odot 2012$ Kowsar Corp. All rights reserved.

- Implication for health policy/practice/research/medical education:

Infections caused by methicillin resistant Staphylocococcus aureus (MRSA) in medical settings are common. In the 1990's, there has been an increase in skin and soft tissue infections due to MRSA strains originating from outside of the medical environment. These infections have been shown to occur mostly in healthy, young individuals. Due to the rise of MRSA, it is necessary to identify new therapeutic solutions to eradicate the pathogen and investigating unique metabolic pathways in the pathogen shows promise in potentially identifying new drug targets.

Please cite this paper as:

Ikawa Wakabayashi JY, Cheng DW. PerR-Mediated Oxidative Stress Response in Staphylococcus aureus. Jundishapur J Microbiol. 2012;5(3):443-9. DOI: 10.5812/jjm.2460

\section{Introduction}

Staphylococcus aureus is a Gram-positive human pathogen, capable of causing a wide range of infections from local infections to metastatic infections in healthy and in immunodeficient patients. Minor skin infections include pimples, impetigo, boils, cellulitis, folliculitis, carbuncles, scalded skin syndrome, and abscesses (1). Serious diseases are pneuomonia, meningitis, osteomyelitis, endocarditis, toxic shock syndrome, chest pain, bacteremia, and sepsis (2-4). Long-term efforts to control this disease worldwide

${ }^{*}$ Corresponding author: Davis W. Cheng, Department of Biology, California State University, Fresno, California, USA 93740. Tel: +1-5592784184, Fax: +15592783963,E-mail: dcheng@csufresno.edu

DOI:10.5812/jjm.2460

Copyright @2012 Kowsar Corp. All rights reserved. have been invested, but all efforts have not been fully effective to control this disease. Recent studies have provided experimental clues of development of some potential strategies to deal with this problem. One of the most significant advancements in this area is the discovery of the transcriptional regulation as the most efficient defense mechanism against oxidative stress due to reactive oxygen species (ROS) in S. aureus resulting from the attack by host phagocytes upon infection (5). Current evidence has shown that stress-responsive genes are activated as part of the cellular defense mechanism where transcriptional regulators sense ROS in many bacteria $(3,6)$. One of these stress-responsive proteins is a major transcriptional regulator PerR (peroxide responsive repressor), which regulates oxidative stress response and mediates the repression of its own gene and other genes on its regulon (7). In this paper, we will analyze 
and discuss the potential transcriptional regulation mechanisms of PerR and its regulon and how PerR regulation influences virulence in S. aureus.

\section{S. aureus Infection and Emergence of MRSA}

This pathogen is known to be the leading cause of nosocomial infections due to methicillin resistant strains of $S$. aureus (MRSA), often causing postsurgical wound infections (1). Indeed, an increase in MRSA strains has become a major public health problem (8). It was proposed previously that MRSA was typically originated from hospital sources where the patient had been hospitalized or a person was in contact with a hospitalized patient (9-12). A recent study further provided evidence that only a few critical genetic changes existed among the genomes of $10 \mathrm{~S}$. aureus isolates collected across the U.S., suggesting that a single MRSA strain was originated through point mutations that then led to the variation of several isolates (13). Probably, some nosocomial cases of MRSA are potentially due to the isolates originating from community reservoirs $(5,14)$.

\section{Significance of Oxidative Stress for Sur- vival of $S$. aureus}

The host immune system responds to the phagocytic attack of the pathogen during the initial stages of infection by S. aureus. These phagocytes include neutrophils and macrophages that migrate to the site of infection to engulf the pathogen and ultimately kill it in the phagolysosomal membrane (15). Subsequent to these reactions, the superoxide anion, hypochlorous acid and nitric oxide can react with other molecules to produce other ROS, such as hydrogen peroxide, peroxynitrite, and hypochlorous acid (15). In fact, production of various types of ROS is a common mechanism in many biological systems and is considered a general inducer of oxidative stress-related gene expression that leads to programmed cell death through several specified signal transduction pathways (16-18). Similarly, in order for the pathogen to protect itself against the ROS from host, genes involved in the regulation of molecular counter-defenses against ROS are subsequently expressed in the pathogen cells. In conjunction with the expression of the pathogen protecting itself against ROS, other genes that produce virulence factors are coordinately expressed to aid in the infection of the host (7). The gene expression of many virulence factors is controlled by regulatory systems with various transcription factors $(5,8,15,16,18,19)$. Several global regulators have been identified to be involved in the process of producing virulence factors, along with oxidative stress response in various pathogens including $S$. aureus $(20,21)$. One of the major transcription factors associated with oxidative stress response and $S$. aureus virulence is $\operatorname{PerR}(22)$.

\section{PerR as a Universal Regulator}

The perR gene found in S. aureus is 447 base pairs in length that encodes a functional protein with 148 amino acids (Gene ID 2859896, NCBI). In S. aureus, PerR has been shown to control an oxidative stress regulon, regulate iron storage proteins, and influence expression of virulence factors (22). This protein in S. aureus is a Mn (II) repressor and the PerR regulon responds to high levels of Fe (II) (23). One of the metal binding sites in PerR is involved in DNA binding and the second site involves zinc ( $\mathrm{Zn}$ ) coordination for the protein structure $(24,25)$. It appears that PerR could be a universal regulator that exists in various bacterial pathogens $(26,27)$. Our phylogenetic analysis revealed high ami-

Figure 1. Phylogenetic Tree Analysis Based Upon the Fast Minimum Evolution Algorithm.

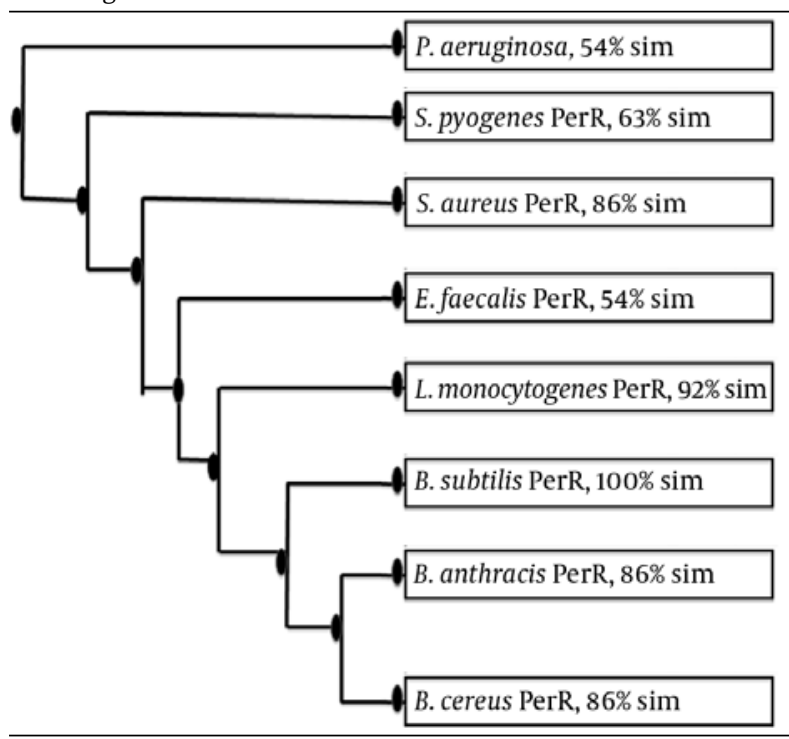

The maximum sequence difference was set to 0.85 . The evolutionary distance model used was the Grishin model. The PerR proteins from the different pathogens and P. aeruginosa Fur share a common evolutionary history. The $\%$ amino acid sim are included where the sequences were compared against B. subtilis

Abbreviation: sim : Amino acid sequence similarity

\begin{tabular}{|c|c|c|}
\hline Gene & PerR Box & $\begin{array}{l}\text { PerR Box Location From } \\
-35 \text { and }-10 \text { Elements }\end{array}$ \\
\hline katA & ATTATAATTATTATA--A-- & Upstream of -10 \\
\hline ftn & ATTATAATTATTATT--AT- & Upstream of -10 \\
\hline$b c p$ & --TAAAATTATTATA--AT- & Upstream of -10 \\
\hline fur & -TTTTAATTATTAGT-A-- & Upstream of -10 \\
\hline $\operatorname{ahpC}$ & ATTAGAATTATTATA--ATT & Upstream of -10 \\
\hline $\operatorname{tr} x B$ & ATAATTATTATTATT---- & Upstream of -10 \\
\hline $\operatorname{mrgA}$ & ATTAGAATTATTATA--AT- & Between -35 and -10 \\
\hline perR & ATAATAATTATTATA---- & Upstream of -10 \\
\hline
\end{tabular}

$\mathrm{a}$ The green nucleotides are conserved among the PerR boxes. PerR boxes for regulon genes were analyzed using online sequence alignment tools (JCMI and NCBI). S. aureus subsp. aureus MRSA252 was used for comparison. 
no acid sequence homologies (54-100 \%) in several representative bacterial pathogens (Figure 1), where PerR amino acid sequence alignment of Bacillus subtilis PerR (GenBank Accession NP_388753.1) v.s other Gram-positive bacteria PerR strains showed that several pathogens (GenBank Accession YP_004876448.1 for B. anthracis, GenBank Accession AAN61567.1 for B. cereus, YP_003464888.1 for Listeria monocytogenes, AAY63967.1 for Enterococcus faecalis, ABF37117.1 for Streptococcus pyogenes, and YP_041326.1 for S. aureus) contain PerR with similarity above $60 \%$. S. aureus PerR has a sequence similarity of $86 \%$ to B. subtilis PerR and $54 \%$ to $P$. aeruginosa Fur. In fact, PerR and PerR homologues have been found in more Gram positive bacteria and less so in Gram negative bacteria $(26,27)$.

\section{3D Structure and Function of PerR Pro- tein}

PerR, like other Fur homologues, is a dimeric protein consisting of two monomers, each of which contains two metal ions (19). For the Zn binding sites on B. subtilis PerR, the $\mathrm{Zn}$ ion is bound by four cysteinates (19). Upon our amino acid sequence alignment analysis of $B$. subtilis PerR, S. aureus PerR, and P. aeruginosa Fur, the four con- served cysteines are observed between $B$. subtilis PerR and S. aureus at similar positions and strictly organized as two Cys-(Xaa) $)_{2}$ motifs with Cys-96, Cys-99, Cys-136, and Cys-139 for B. subtilis PerR and S. aureus PerR(Figure 2). It has been found that the $\mathrm{Zn}(\mathrm{Cys})_{4}$ site in PerR-Zn joins the three S3, S4, and S5 beta-strands of each monomer to form the two dimmers, locking together the three strands of the betasheet (28). Due to the presence of the four cysteines in the $S$. aureus PerR, it is likely that these PerR proteins also contain a $\mathrm{Zn}(\mathrm{Cys})_{4}$ site. This $\mathrm{Zn}(\mathrm{Cys})_{4}$ site may be a unique feature of the PerR and PerR homologue regulators (4).

It was speculated that the metal binding to the His-37 causes the N-terminal domain to move towards the $\mathrm{C}$ terminus which is the resultant conformational change capable of PerR binding to DNA (2). These residues may form a shape of an asymmetrical pyramid with the His93 occupying the apical position and His-37, Asp-85, His-91 and Asp-104 forming the pyramid base for binding regulatory metals such as $\mathrm{Fe}(\mathrm{II})$ or Mn(II) ligands necessary for in vivo PerR repressor function in B. subtilis (29). Our further analysis of the $S$. aureus PerR structure show the $\mathrm{Zn}$ binding site consists of the four cysteines involved in the protein coordination (Figure $3 A$ and $3 B$ ). The $\mathrm{Mn}(\mathrm{II})$ bind-

\begin{tabular}{lll}
\hline Table 2. A List of PerR Regulon & \\
\hline Gene & Gene Product & Function \\
\hline$k a t A$ & Catalase & Catalase activity, oxidative stress \\
$f t n$ & Ferritin & Iron storage proteins \\
$b c p$ & Thiol peroxidase & Antioxidant activity, oxidative stress \\
$f u r$ & Ferric uptake regulator & Transcriptional regulator, iron transport and metabolism \\
$a h p C$ & Akyl hydroperoxide reductase & Peroxiredoxin activity, oxidative stress \\
$t r x B$ & Thioredoxin reductase & Thioredoxin-disulfide reductase activity, oxidation-reduction process \\
$m r g A$ & Ferritin-like Dps homologue & Ferric ion binding, metalloregulation DNA-binding stress protein \\
perR & Perxoide-responsive repressor & Transcriptional regulator \\
\hline
\end{tabular}

\begin{tabular}{|c|c|c|c|c|c|}
\hline Primary Locus $^{\text {a }}$ & Common Name ${ }^{a}$ & $\begin{array}{l}\text { Gene Length } \\
\text { (bps) }\end{array}$ & $\begin{array}{l}\text { Codons, } \\
\text { No. }\end{array}$ & Protein Function & $\begin{array}{l}\text { \% Identity to } \\
\text { PerR Box }{ }^{b}, \%\end{array}$ \\
\hline NWMN 0815 & $\begin{array}{l}\text { conserved hypothetical } \\
\text { protein }\end{array}$ & 1155 & 385 & cellular component & 94 \\
\hline NWMN 0731 & TPR domain protein & 1440 & 480 & unknown & 88 \\
\hline NWMN 0515 & $\begin{array}{l}\text { polysaccharide biosynthesis } \\
\text { protein CapD }\end{array}$ & 966 & 322 & coenzyme binding & 82 \\
\hline NMWN 2256 & $\begin{array}{l}\text { teicoplanin resistance associ- } \\
\text { ated protein B }\end{array}$ & 1209 & 403 & $\begin{array}{l}\text { drug transmembrane transport- } \\
\text { er activity }\end{array}$ & 82 \\
\hline NWMN 0892 & serine protease HtrA & 2325 & 775 & $\begin{array}{l}\text { protein fate: degradation of pro- } \\
\text { teins, peptides, and glycoproteins }\end{array}$ & 76 \\
\hline NWMN 1257 & exonuclease SbcD & 1122 & 775 & exonuclease activity & 76 \\
\hline NWMN 1754 & $\begin{array}{l}\text { D-isomer specific 2-hydroxyacid } \\
\text { dehydrogenase family protein }\end{array}$ & 951 & 317 & NAD binding & 76 \\
\hline NWMN 2397 & fibronectin binding & 2034 & 678 & $\begin{array}{l}\text { cellular component; biological } \\
\text { process }\end{array}$ & 76 \\
\hline
\end{tabular}

${ }^{a}$ Comprehensive Microbial Resource

${ }^{\mathrm{b}}$ NCBI BLAST with ATTATAATTATTATTAT as PerR Box (Morrissey, et al., 2004) 
Figure 2. Clustal Sequence Alignment of B. subtilus PerR, S. aureus PerR and P. aeruginosa Fur.

$$
\begin{aligned}
& \text { Bs_PerR } \\
& \text { Sa_PerR } \\
& \text { Pa_Fur }
\end{aligned}
$$

$$
\begin{aligned}
& \text { Bs_PerR } \\
& \text { Sa_PerR } \\
& \text { Pa_Fur }
\end{aligned}
$$

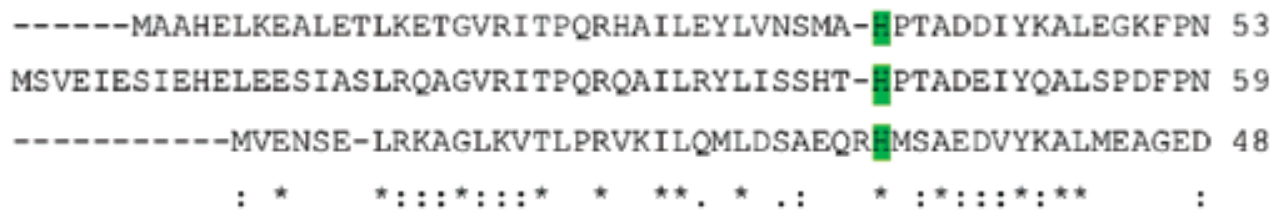

The GenBank accession numbers of fur gene sequences used in this analysis are NP_388753.1 for B. subtilus PerR, YP_041326.1 for S. aureus PerR and NP_253452.1 for P. aeruginosa, respectively. The yellow highlight indicates the four cysteines needed for $\mathrm{Zn}$ coordination. The green highlight shows the amino acids involved in the metal regulatory site. The blue highlight shows the three conserved amino acids involved in the third putative site.

ing site is located exterior to the protein, between the $\mathrm{N}$ and C-terminal domains. This open area may be the site for hydrogen peroxide recruitment (29). The empty coordination site on $\mathrm{Mn}$ includes four chain carbonyls from Ala-36, Phe-86, Ser-89, and Val-103, a hydroxyl group from Thr-88, and an amino group of Lys-101. The best-fit model for the $B$. subtilis PerR Fe coordination is a similar square based pyramid orientation as the Mn coordination (2). Therefore, the B. subtilis PerR-Zn-Fe has the same pentacoordinate $\mathrm{His}_{3} \mathrm{Asp}_{2}$ environment where both $\mathrm{Mn}(\mathrm{II})$ and
$\mathrm{Fe}(\mathrm{II})$ appear to compete for the same protein binding site (25). The S. aureus PerR is suspected to have a similar coordination pattern.

\section{PerR Regulons and PerR Boxes}

PerR controls the transcription of genes encoding the oxidative stress resistance proteins and also regulates transcription of genes encoding iron storage proteins. In recent years, the putative PerR binding sites have been

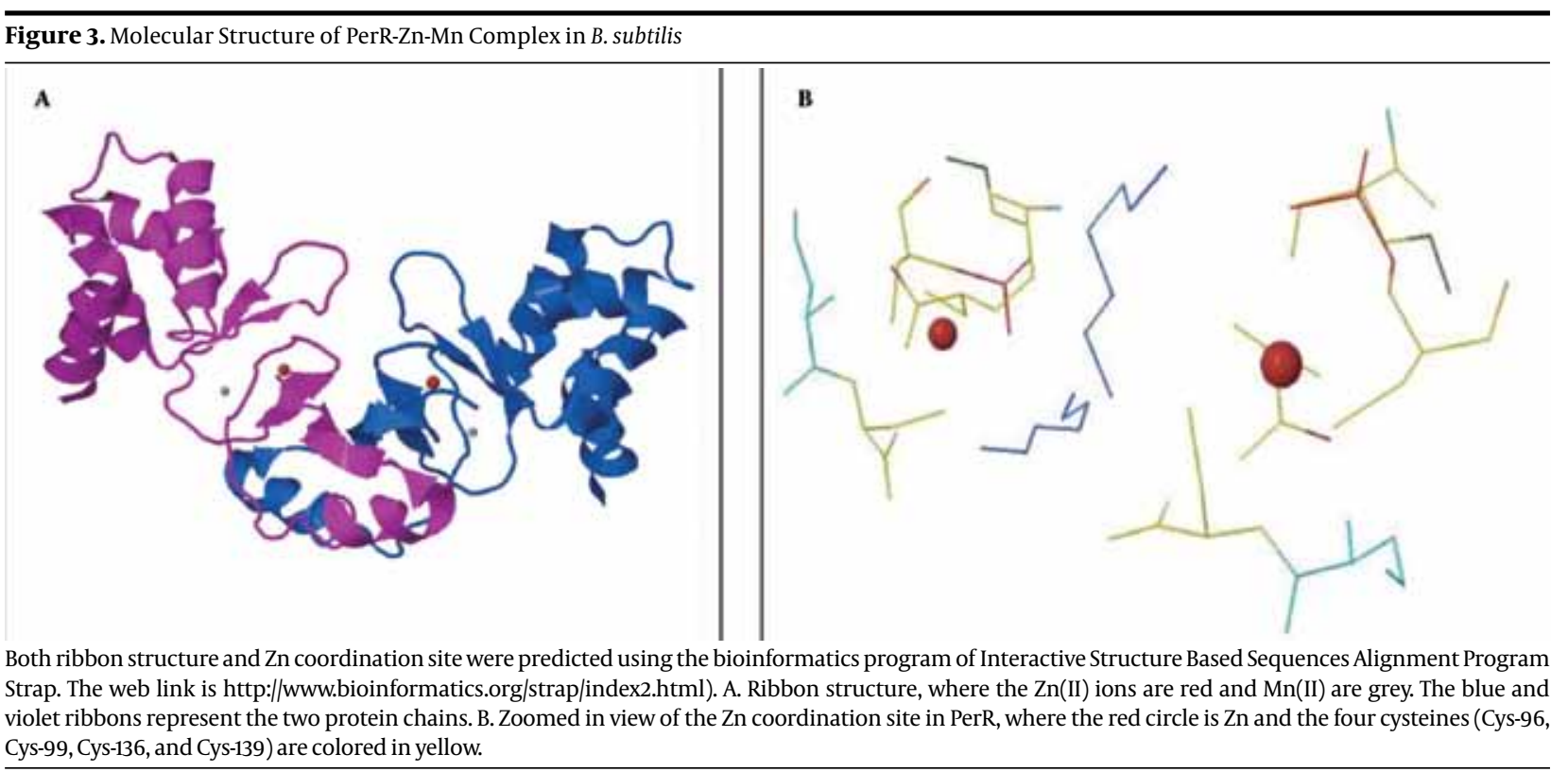




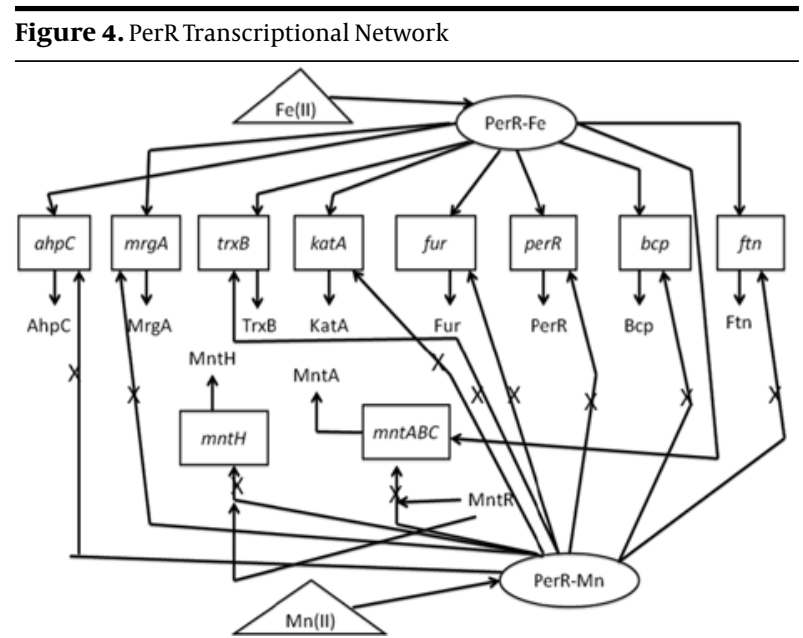

The rectangles are the genes regulated by PerR on the PerR regulon and the Mnt operon. The gene products are in black print. The ovals are the metalloregulators (PerR-Zn-Fe or PerR-Zn-Mn). The triangles are the metal ligands. The $\mathrm{X}$ represents that transcription of that gene is blocked.

determined to identify genes controlled by PerR (22). It has been reported that the PerR binding site for perR, fur, $a h p C$, katA, and $m r g A$ genes are located in their promoter region near the -35 and -10 elements located either upstream from the -10 element or between the -35 and -10 elements as is the case for the $m r g A$ gene, of which the $f t n A$ and $m r g A$ genes contain two PerR boxes each(23). Some of these genes are located on the same regulon as the gene that encodes the PerR protein, such as fur, ftn, perR, trxB, and $b c p(11,22)$.

A putative PerR box was identified at the upstream of the $n f r A$ promoter where 9 of 14 nucleotides (TTCAATTATTAACTT) are the same as the consensus sequence (TATAATTATTATTA) for the PerR box that is responsible for the induced transcription of $n f r A$ by hydrogen peroxide at high concentrations (12). Another PerR box that may regulate genes outside of oxidative stress response and Fe and Mn homeostasis may exist. In S. aureus str. Newman, many similar short sequences were identified with an identity ranging from 42-94\% against the conserved PerR box sequences of ATTATAATTATTATTAT (16). Eight of these short sequences with identities of 76-94\% can be predicted in the coding regions for proteins with known functions except for one where the function is unknown (Table 3).

\section{Oxidative Stress Response by PerR and PerR Regulon in S. aureus}

PerR may function as a major regulator of antioxidant defenses and storage regulator for Fe. PerR regulates a major oxidative stress regulon involved in intracellular thiol-disulfide balance, which include genes encoding for catalase, alkyl hydroperoxide reductase, thioredoxindependent thiol peroxidase (Bcp), and thioredoxin reductase (TrxR) (22). Upon our investigations, these genes may provide reducing power to help eliminate ROS upon induction and help maintain their cellular redox balance, partially due to the presence of low molecular weight thiols $(9,10,30)$. The presence of the putative PerR box near the bcp gene in S. aureus suggests that PerR has a regulatory role in transcription of thioredoxin and thiol peroxidases (28).

Other genes on the PerR regulon involve the Fe storage and Fe homeostasis regulator proteins (Fe storage protein ferritin, Ftn and ferritin-like Dps homologue, MrgA) which auto-regulates the expression of Fur (Fe homeostasis regulator) (22). The direct and indirect control of Fe homeostasis by the PerR regulon may allow $S$. aureus to maintain a non-toxic level of free Fe and redox stability to combat against ROS produced by Fenton reactions.

It is clear that there is a general transcriptional network for PerR, its regulon, and separate mnt operon as we proposed and illustrated in Figure 4. Within this network, the PerR protein is a Mn (II) repressor where mntABC transport gene is shut off in the presence of PerR-Mn and activated in the presence of PerR-Fe. The PerR regulon is induced by responding to high levels of Fe (PerR-Fe) and transcription of its genes are repressed with Mn (PerR$\mathrm{Mn})$. This simplistic network does not account for the complexity of how certain genes such as fur and perR are auto-regulated, how ahpC and katA have compensatory roles in their gene expression, and the bifunctional role of MntR to repress $m n t H$ when Mn (II) concentration is high and induces $m n t A B C$ at low Mn (II) concentration.

\section{Impact of PerR and PerR regulon on S. au- reus Virulence}

The PerR regulon contains at least four genes including perR, fur, ahpC and katA in S. aureus. A perR mutant demonstrated a lower degree of virulence in a skin abscess model of infection in mice (22). A fur mutant became less virulent potentially due to its reduced infection in a murine skin abscess model (20). The katA gene encoded catalase has also been considered to be an important virulence determinant in S. aureus due to their higher levels of superoxide dismutase (SOD) and catalase expression detected in clinical studies (21). ahpC compensates for the lack of $k a t A$ where $a h p C$ is expressed at a higher level, however, $a h p C$ and katA do not likely play a role in protection from nasal secretions since similar proteins are found in neutrophils and $S$. aureus reduction by neutrophils was not observed (31). It is possible that S. aureus lacking $a h p C$ and katA is compromised due to the lack of ability to survive under aerobic conditions.

The importance of $a h p C$ and katA expression has been demonstrated for $S$. aureus to withstand harsh environmental conditions such as starvation or desiccation(32). ahpC and katA have demonstrated roles in desiccation tolerance as disrupted mutants $(\triangle a h p C \Delta k a t A)$ showed a desiccation defect (33). Catalase has been shown to be 
crucial for the longevity of starved S. aureus, where survival against ROS would be required (22). Therefore, PerR and its regulon appear to be involved in S. aureus virulence and longevity S. aureus virulence and longevity.

\section{Conclusions and Prospects}

Genetic network analysis is a powerful tool to elucidate the gene-gene and protein-protein interaction involved in S. aureus infections. The PerR binding site exists in the promoter regions of perR, fur, ahpC, katA, and mrgA genes (23). Some of these genes are located on the same regulon as the gene that encodes the PerR protein, such as fur, ftn, perR, trxB, and $b c p(11,22)$. Our analysis has led to an identification of several novel PerR boxes present in S. aureus due to the existence of the consensus sequence ATTATAATTATTATTA at different coordinates throughout its genome. These PerR DNA binding sites for PerR gene regulation suggests a novel role for PerR in mediating stress responses and other cellular processes which potentially contributes to the pathogenic fitness of $S$. aureus surviving in the infected host.

Presently, the oxidative stress responses by PerR and the PerR regulon do play a major role related to the longterm survival of $S$. aureus under unfavorable conditions as desiccation and / or long-term starvation, which has been implicated in nosocomial S. aureus transmission and found to exacerbate the MRSA problem (34). Here, we propose that the oxidative stress resistance proteins are regulated by PerR as a major regulator of antioxidant defenses and storage regulator responsible for the longterm survival of $S$. aureus. These oxidative stress resistance proteins include catalase, alkyl hydroperoxide reductase, thioredoxin-dependent thiol peroxidase (Bcp), and thioredoxin reductase (TrxR).

To date, it appears that PerR may function as a universal regulator for many genes located in the cascade pathway of oxidative stress responses that further activate the expression of virulence genes upon infection of the host, a period, and spread in the communities. As a conclusion, we hypothesize that PerR and its regulon influences the expression of virulence factors by prolonging the pathogen's survival under harsh conditions that may lead to the increased risk of $S$. aureus transmission, infection, and spread of the disease. Therefore, several approaches should be taken to control and eventually minimize the incidences of S. aureus infections. First, several key minerals such as $\mathrm{Mn}, \mathrm{Fe}, \mathrm{Zn}$ could be formulated for medicinal use and optimized for testing in clinical trials; Second, it is possible to develop enzyme inhibitors against the PerR protein and other proteins in the PerR regulon; Third, anti-sense RNA (or RNAi) technology could be developed to control PerR gene expression using PerR box sequences. Consequently, it behooves us to further elucidate the PerR regulatory network in order to identify and characterize unique molecular targets for use in developing therapeutic strategies for eradicating S. aureus.

\section{Acknowledgments}

None declared.

\section{Financial Disclosure}

None declared.

\section{Funding/Support}

The authors acknowledge the National Institute on Minority Health and Health Disparities in the United States of America for their financial support to this research work.

\section{References}

1. Lee AS, Huttner B, Harbarth S. Control of Methicillin-resistant Staphylococcus aureus. Infec Dis Clin North AM. 2011;25(1):155-79.

2. Jacquamet L, Traore DA, Ferrer JL, Proux O, Testemale D, Hazemann JL, et al. Structural characterization of the active form of PerR: insights into the metal-induced activation of PerR and Fur proteins for DNA binding. Mol Microbiol. 2009;73(1):20-31.

3. Newberry KJ, Fuangthong M, Panmanee W, Mongkolsuk S, Brennan RG. Structural mechanism of organic hydroperoxide induction of the transcription regulator OhrR. Mol Cell. 2007;28(4):652-64.

4. Traore DA, El Ghazouani A, Ilango S, Dupuy J, Jacquamet L, Ferrer JL, et al. Crystal structure of the apo-PerR-Zn protein from Bacillus subtilis. Mol Microbiol. 2006;61(5):1211-9.

5. Deresinski S. Methicillin-resistant Staphylococcus aureus: an evolutionary, epidemiologic, and therapeutic odyssey. Clin Infect Dis. 2005;40(4):562-73.

6. Helmann JD, Wu MF, Gaballa A, Kobel PA, Morshedi MM, Fawcett $\mathrm{P}$, et al. The global transcriptional response of Bacillus subtilis to peroxide stress is coordinated by three transcription factors. $J$ Bacteriol. 2003;185(1):243-53.

7. Somerville GA, Proctor RA. At the Crossroads of Bacterial Metabolism and Virulence Factor Synthesis in Staphylococci. Microbiol Mol Biol R. 2009;73(2):233-48.

8. Chambers HF. The changing epidemiology of Staphylococcus aureus? Emerg Infect Dis. 2001;7(2):178-82.

9. Newton GL, Leung SS, Wakabayashi JI, Rawat M, Fahey RC. The DinB superfamily includes novel mycothiol, bacillithiol, and glutathione S-transferases. Biochemistry. 2011;50(49):10751-60.

10. Sharma SV, Jothivasan VK, Newton GL, Upton H, Wakabayashi JI, Kane MG, et al. Chemical and Chemoenzymatic syntheses of bacillithiol: a unique low-molecular-weight thiol amongst low $\mathrm{G}+\mathrm{C}$ Gram-positive bacteria. Angew Chem Int Ed Engl. 2011;50(31):7101-4.

11. Soutourina O, Dubrac S, Poupel O, Msadek T, Martin-Verstraete I. The pleiotropic CymR regulator of Staphylococcus aureus plays an important role in virulence and stress response. PLoS Pathog. 2010;6(5):e1000894.

12. Streker K, Freiberg C, Labischinski H, Hacker J, Ohlsen K. Staphylococcus aureus NfrA (SA0367) Is a Flavin Mononucleotide-Dependent NADPH Oxidase Involved in Oxidative Stress Response.J Bacteriol. 2005;187(7):2249-56.

13. Nicolle L. Community-acquired MRSA: a practitioner's guide. $\mathrm{Ca}$ nadian Med Associat J. 2006;175(2):145.

14. Kader O, Ebid S, Mostafa N, El Sayed S, Ghazal A. Detection of community acquired methicillin resistance Staphylococcus aureus among Staphylococus aureus isolates. J AM Sci. 2011;7(1):1109-17.

15. Clements MO, Foster SJ. Stress resistance in Staphylococcus aureus. Trends Microbiol.1999;7(11):458-62.

16. Cheng DW, Jiang Y, Shalev A, Kowluru R, Crook ED, Singh LP. An analysis of high glucose and glucosamine-induced gene expression and oxidative stress in renal mesangial cells. Arch Physio Biochem. 2006;112(4-5):189-218.

17. Singh LP, Cheng DW, Kowluru R, Levi E, Jiang Y. Hexosamine in- 
duction of oxidative stress, hypertrophy and laminin expression in renal mesangial cells: effect of the anti-oxidant alpha-lipoic acid. Cell Biochem Funct. 2007;25(5):537-50.

18. Singh LP, Jiang Y, Cheng DW. Proteomic identification of 14-33zeta as an adapter for IGF-1 and Akt/GSK-3beta signaling and survival of renal mesangial cells. Int J Biol Sci. 2007;3(1):27-39.

19. Herbig AF, Helmann JD. Roles of metal ions and hydrogen peroxide in modulating the interaction of the Bacillus subtilis PerR peroxide regulon repressor with operator DNA. Mol Microbiol. 2001;41(4):849-59.

20. Horsburgh MJ, Ingham E, Foster SJ. In Staphylococcus aureus, fur is an interactive regulator with PerR, contributes to virulence, and Is necessary for oxidative stress resistance through positive regulation of catalase and iron homeostasis. J Bacteriol. 2001;183(2):468-75.

21. Kanafani H, Martin SE. Catalase and superoxide dismutase activities in virulent and nonvirulent Staphylococcus aureus isolates. JClin Microbiol.1985;21(4):607-10.

22. Horsburgh MJ, Clements MO, Crossley H, Ingham E, Foster SJ. PerR controls oxidative stress resistance and iron storage proteins and is required for virulence in Staphylococcus aureus. Infect Immun. 2001;69(6):3744-54.

23. Morrissey JA, Cockayne A, Brummell K, Williams P. The Staphylococcal Ferritins Are Differentially Regulated in Response to Iron and Manganese and via PerR and Fur. Infect Immun. 2004;72(2):972-9.

24. Giedroc DP. Hydrogen peroxide sensing in Bacillus subtilis: it is all about the (metallo)regulator. Mol Microbiol. 2009;73(1):1-4

25. Lee JW, Helmann JD. Biochemical characterization of the structural Zn2+ site in the Bacillus subtilis peroxide sensor PerR. J Biol Chem. 2006;281(33):23567-78.

26. Faulkner MJ, Helmann JD. Peroxide stress elicits adaptive changes in bacterial metal ion homeostasis. Antioxid Redox Signal.
2011;15(1):175-89.

27. Lee JW, Helmann JD. Functional specialization within the Fur family of metalloregulators. Biometals. 2007;20(3-4):485-99.

28. Jeong W, Cha MK, Kim IH. Thioredoxin-dependent hydroperoxide peroxidase activity of bacterioferritin comigratory protein (BCP) as a new member of the thiol-specific antioxidant protein (TSA)/Alkyl hydroperoxide peroxidase C (AhpC) family. J Biol Chem. 2000;275(4):2924-30.

29. Lee J-W, Helmann JD. The PerR transcription factor senses $\mathrm{H} 2 \mathrm{O} 2$ by metal-catalysed histidine oxidation. Nature. [10.1038/nature04537]. 2006;440(7082):363-7.

30. Gaballa A, Newton GL, Antelmann H, Parsonage D, Upton $\mathrm{H}$, Rawat $\mathrm{M}$, et al. Biosynthesis and functions of bacillithiol, a major low-molecular-weight thiol in Bacilli. Proc Natl Acad Sci U S A. 2010;107(14):6482-6.

31. Cosgrove K, Coutts G, Jonsson IM, Tarkowski A, Kokai-Kun JF, Mond JJ, et al. Catalase (KatA) and alkyl hydroperoxide reductase (AhpC) have compensatory roles in peroxide stress resistance and are required for survival, persistence, and nasal colonization in Staphylococcus aureus. J Bacteriol. 2007;189(3):1025-35.

32. Beard-Pegler MA, Stubbs E, Vickery AM. Observations on the resistance to drying of staphylococcal strains. J Med Microbiol. 1988;26(4):251-5.

33. Chaibenjawong P, Foster SJ. Desiccation tolerance in Staphylococcus aureus. Arch Microbiol. 2011;193(2):125-35.

34. Duckworth GJ, Jordens JZ. Adherence and survival properties of an epidemic methicillin-resistant strain of Staphylococcus aureus compared with those of methicillin-sensitive strains. J Med Microbiol.1990;32(3):195-200.

35. Horsburgh MJ, Clements MO, Crossley H, Ingham E, Foster SJ. PerR Controls Oxidative Stress Resistance and Iron Storage Proteins and Is Required for Virulence in Staphylococcus aureus. Infect Immun. 2001;69(6):3744-54. 